\title{
Epidemiology of pertussis in Casablanca (Morocco): contribution of conventional and molecular diagnosis tools
}

Khalid Katfy ${ }^{1,2,7^{*}}$, Nicole Guiso ${ }^{3}$, Idrissa Diawara ${ }^{1,2}$, Khalid Zerouali ${ }^{1,2}$, Bouchra Slaoui $^{4}$, Zineb Jouhadi ${ }^{5}$, Abdelhadi Zineddine ${ }^{6}$, Houria Belabbes ${ }^{1,2}$ and Naima Elmdaghri, ${ }^{1,2}$

\begin{abstract}
Background: Pertussis, a vaccine preventable disease, is still responsible of significant morbidity and mortality around the world, mostly in newborns. The aim of the present study was (1) to introduce pertussis surveillance in the major pediatric hospital of Casablanca (2) to analyze the prevalence of pertussis among children under 14 years of age and their entourage in Casablanca, Morocco.

Methods: This is a prospective and non-case controlled study, including children suspected of Pertussis admitted at the Abderrahim Harouchi Pediatric Hospital in Casablanca, from January 2013 to June 2015. Nasopharyngeal samples were obtained for Bordetella spp. culture and Real time PCR detection (RT-PCR) with specific primers of Bordetella spp., B. pertussis, B. parapertussis and B. holmesii. The detection of Bordetella spp. was also performed in some household contacts of the children suspected of pertussis.

Results: During the 2.5-years period, a total of 282 samples were collected from hospitalized children (156) and in some of their contacts (126). Among 156 samples from the children (from whom 57\% were under 2 month of age), Bordetella DNA was detected in 61\% (96/156) by RT-PCR. Among these positive samples, $91.7 \%$ (88/96)

corresponded to B. pertussis DNA. Furthermore, in 39.5\% (38/96) of the Bordetella positive samples, B. holmesii DNA was also detected. B. parapertussis DNA was detected in only one sample (1/156). Out of the 156 samples collected from the hospitalized children, only 48 were tested by culture, and 4 B. pertussis were isolated (8.3\%). Among the 126 samples from the contacts of the children, mostly mothers (115 cases), Bordetella DNA was detected in 47\% (59/126), 90\% (53/59) being B. pertussis DNA. Moreover, B. holmesii DNA was also detected in 18.6\% (11/59) of the Bordetella positive samples, and coexistence of B. pertussis and B. holmesii DNA in 36.5\% (35/96). Two B. pertussis were isolated by culture performed on 43 samples of the contacts of the children (4.6\%).

Conclusions: This study highlights the circulation of B. pertussis but also of B. holmesii in Casablanca-Morocco with a high proportion of co-infections $B$. holmesii/B. pertussis in infants and their mothers, indicate that infection of non-vaccinated infants could be more associated with young parents. Moreover, the RT- PCR provides a sensitive and specific diagnosis of $B$. pertussis infections and distinguishes it from other Bordetella species, and is therefore suitable for implementation in the diagnostic laboratory.
\end{abstract}

Keywords: Pertussis, B. Pertussis, B. Parapertussis, B. Holmesii, Pertussis toxin gene, Pertussis whole cell vaccine, RT-PCR

\footnotetext{
* Correspondence: khalidkatfy@hotmail.com

'Laboratoire de Microbiologie, Faculté de Médecine et de Pharmacie, Hassan

II University of Casablanca, B, P 5696 Casablanca, Morocco

${ }^{2}$ Service de Microbiologie, CHU Ibn Rochd, B, P 2698 Casablanca, Morocco

Full list of author information is available at the end of the article
} International License (http://creativecommons.org/licenses/by/4.0/), which permits unrestricted use, distribution, and reproduction in any medium, provided you give appropriate credit to the original author(s) and the source, provide a link to the Creative Commons license, and indicate if changes were made. The Creative Commons Public Domain Dedication waiver (http://creativecommons.org/publicdomain/zero/1.0/) applies to the data made available in this article, unless otherwise stated. 


\section{Background}

Bordetella pertussis is the agent of whooping cough (pertussis), an endemic illness responsible for significant morbidity and mortality, especially in infants and children [1] Worldwide, there are an estimated 16 millions cases of pertussis, 95\% of which occur in developing countries, resulting in about 195,000 child deaths per year [1].The epidemiology of pertussis, which is a cyclical disease, is varying from regions to regions because of differences in the type of vaccine used, type of vaccine strategy, and differences in surveillance or lack of surveillance.

Pertussis vaccination has been introduced in almost all vaccination programs throughout the world and has reduced significantly the morbidity and the mortality related to the disease. In the context of highcoverage vaccination coverage, the reported cases are predominantly registered among children $<1$ year-old with a high proportion among infants $<3$ months-old (Ref). Adults are considered as the main reservoir of transmission of the disease to unvaccinated young children and several immunization strategies have been proposed in order to prevent cases among young children [2].

In Morocco, Pertussis vaccination [pertussis whole cell vaccine $(\mathrm{wP})$ in combination with diphtheria and tetanus toxoids (DTwP)] was introduced by the national immunization program (NIP) in the early 1980's to prevent pertussis, allowed the reduction of the incidence of the disease from almost 15,000 cases per year in 1980 to less than 100 cases per year by 2011 [3].

Currently, in Morocco, the vaccination strategy includes a primary vaccination at 2,3 and 4 months of age and two boosters at 18 months of age and 5 years of age. The vaccine is provided through UNICEF. The vaccine pertussis vaccine coverage exceeds $95 \%$ at the age of 24 months [4]. Starting in January 2012, a significant increase in the number of cases was registered: during one month, in March 2012, eighty cases were identified and lab-confirmed in the locality of Sale, Morocco. Subsequently, numerous cases were reported across the country, and in August of the same year, another outbreak occurred in Casablanca with more than 130 clinically diagnosed cases hospitalized in the Abderrahim Harouchi children hospital [5].

However, pertussis cannot be confirmed based only on a clinical diagnosis because of the different presentations of the disease in non-vaccinated newborns, partially vaccinated children and adolescents and adults whose immunity waned with time [6].Therefore, laboratory tests are needed for confirmation of the diagnosis in those patients showing suggestive clinical signs and symptoms, or those with a history of exposure to infected patients [7].
Currently available $B$. pertussis diagnostic methods include direct diagnosis such as culture, and nucleic acid amplification assays and indirect diagnosis such as the detection of anti-pertussis toxin antibodies [8]. Culture is specific and sensitive in infants and young children but less in adolescents and adults, coming late after the onset of cough as previously reported by Nakamura et al. 2011 [9]. PCR assays are faster, highly sensitive, and their use in epidemiological surveillance has increased recently to measure the impact of the disease in the community, and to improve studies of vaccine efficacy. However, the routinely real time PCR (RT-PCR) used during the last decade, although very sensitive, is a Bordetella PCR. It is based on Insertion Sequence (IS) 481. This RT-PCR is very sensitive because the IS481 is present in multicopies on the chromosome of $B$. pertussis [10], however it is no more specific of $B$. pertussis since the target (IS481) is also harbored by the chromosome of $B$. holmesii and some B. bronchiseptica isolates [11]. B. holmesii was first described as the agent of bacteremia in asplenic patients [12]. Later on it was first detected in respiratory samples during pertussis outbreaks [13]. It is not yet known whether B. holmesii is a real pathogen or an opportunistic bacterium [14]. However, the recent increase of its detection in respiratory samples is mostly due to the use of the RT-PCR based on the IS481 target as shown in several studies [14-16]. Additional specific RT-PCRs are then needed to determine the nature of the Bordetella species responsible of pertussis.

The aim of the present study was (1) to introduce pertussis surveillance in the major pediatric hospital of Casablanca (2) to analyze the prevalence of pertussis among children under 14 years of age and their entourage in Casablanca, Morocco.

\section{Methods \\ Patients}

This is a prospective and non-case controlled study including all patients younger than 14 years of age admitted to the Abderrahim Harouchi Pediatric Hospital in Casablanca, Morocco, from January 2013 to June 2015and meet the case definition in accordance with the criteria defined by the WHO [3].

Clinical and epidemiological features of each patient were recorded by a physician, including age, symptoms (coughing, paroxysms, respiratory distress, whoops, apnea, cyanosis, seizure, vomiting), days from the onset of symptoms, and time elapsed until the sample was collected and sent to the laboratory. Surveillance of pertussis has been extended to the household contacts to detect index cases and secondary cases and to prevent the spread of the disease. Duplicates of each sample are excluded from the analysis. 


\section{Samples}

Infants (< 12 months) were sampled by nasopharyngeal aspiration using a small catheter inserted through the nostril into the nasopharynx, and flushed with sterile saline. Older children (> 12 months) and adults were sampled by nasopharyngeal swabs which were then placed into a screw cap tube with transport medium (Amies Swab ref. - 300,281 provider DELTALAB).

Nasopharyngeal samples obtained from hospitalized patients, and household contacts were sent to the microbiology laboratory at Ibn Rochd University Hospital in Casablanca at room temperature, accompanied by a fact sheet with all the clinical and socio-demographic indications. All samples were analyzed after an informed consent signed by the parents or caregivers of the children. The research of Bordetella was performed also in hospitalized childrens' contacts after their consent.

\section{Bacterial culture}

The samples were cultured into a fresh Bordet Gengou agar or Regan Low medium (Difco Laboratories, Detroit, USA) prepared in our laboratory, which included $15 \%$ of defibrinated horse's blood and $40 \mathrm{mg} / \mathrm{L}$ of cephalexin (Selective supplement Bordetella, Oxoïd-Unipath, Dardilly, France). The growth media used for bacterial culture were validated using $B$. pertussis (CIP 8132), B. parapertussis (CIP 12822) and B. holmesii (CIP104394) reference strains.

They were incubated at $35 \pm 2{ }^{\circ} \mathrm{C}$ in a moist atmosphere and maintained for three to ten days under aerobic conditions. Colonies were identified based on characteristic morphology, Gram stain, catalase and oxidase testing and by using the API 20NE system (bioMérieux, Marcy L'Etoile, France). The colonies suspected to be Bordetella spp. were tested by molecular tools as described below.

\section{DNA extraction}

Nucleic acids were extracted from submitted specimens or bacterial suspensions using a commercial kit (High Pure Template Preparation Kit, Roche Applied Science, Mannheim, Germany) according to the manufacturer's instructions. The DNA was eluted into a $100-\mu \mathrm{L}$ volume. The obtained DNA was assayed immediately or stored at $-20{ }^{\circ} \mathrm{C}$ until use. Concerning the reference strains B. pertussis CIP 8132, B. parapertussis CIP 12822, and B. holmesii CIP 104394, genomic DNA was extracted using DNeasy Tissue Kit (QIAGEN, Hilden, Germany) according to the manufacturer's protocol.DNA were stored at $-20^{\circ} \mathrm{C}$ until required for analysis.

\section{RT- PCR amplification}

Detection of Bordetella spp. and Bordetella parapertussis DNA was performed using Real time PCR (RT-PCR) assays and $\operatorname{TaqMan}^{\odot}$ technology for the amplification of the insertion elements IS 481 (Bordetella spp), IS1001 (B. parapertussis)as described by Riffelmann et al. 2005 [10].

Since IS 481 target is detecting B. pertussis as well as B. holmesii DNA, we performed, on all positive IS 481 samples, two other specific RT-PCR, one specific of B. pertussis $(p t x A-P r)$ and one specific of $\mathrm{B}$. Holmesii (h-IS1001) in order to confirm the diagnosis of $B$. pertussis and/or B. holmesii [17, 18]. Negative notemplate controls were included in each run. The DNA of $B$. pertussis (Tohama), B. parapertussis (12822) and B. holmesii (104394) were used as positive controls. DNA was amplified with the CFX96 ${ }^{\circ}$ thermal real-time PCR System (Biorad).Potential inhibitory activity was assessed by amplifying human gene sequences RnaseP. A positive result was defined as a cycle threshold $(\mathrm{Ct})$ value below 38 cycles. $\mathrm{Ct}$ values of $>38$ or no detection ( $\mathrm{Ct}$ value of zero) were considered negative.

\section{Quality controls}

The RT- PCR assays were evaluated through an external quality control program managed by the Bordetella National Reference Centre - Institut Pasteur, Paris, France [19].

\section{Statistical analysis}

Data entry was performed using WHONET 5.6 software. Analyzes were performed using Epi info (CDC, Atlanta, Georgia) and Microsoft Excel. Qualitative variable analysis was performed by nonparametric tests: The chi square test or Fisher's exact test. For quantitative variables the Student's test (T) was used. A difference is considered statistically significant if $p$-value $<0.05$.

\section{Results}

During thirty months of surveillance, a total of 282 nasopharyngeal samples were collected from patients with clinical suspicion of pertussis disease and some of their household contacts (156 infants/126 contacts) and were analyzed in the microbiology lab. The majority of patients $85 \%$ (132/156 infants) were admitted in the Pediatric Respiratory Diseases ward and 15\% (24/156) were hospitalized in intensive care unit indicating the severity of the disease.

The monthly distribution of pertussis cases was not homogeneous. The majority of cases were reported during two periods: $54 \%$ (84/156) of cases between April and June, 39\% (61/156) of cases between September and November.

Hospitalized children were below 5 years of age, with an average of $60 \pm 10$ days and $57 \%$ (89/156) were under 2 months of age. The average duration of hospitalization 
was $4 \pm 1$ days, and for cases hospitalized in intensive care, the average was $28 \pm 7$ days with the minimum duration to 21 days and the maximum to 35 days according to associated complications. Among the 126 contacts, 115 were children's mothers; four siblings aged 3 to 6 years and 7 grand-parents. As for the children's mothers the average age was $26 \pm 4.77$ years of age.

Of the 282 samples collected from all cases, culture was performed only for 91 samples: 48 from hospitalized children and 43 from their contacts. B. pertussis was isolated in $8.3 \%(4 / 48)$ and $4.6 \%(2 / 43)$ of the samples, respectively. We collected only $6 \mathrm{~B}$. pertussis isolates mainly because most of the time the patients were already treated with macrolides $(39 / 48$, i.e. $81.3 \%$ ) or coming late after the beginning of the cough $(27 / 48$, i.e. $56.3 \%$ ) or because the medium was not available or the laboratory is not working all days at the hospital. All samples were tested by RT-PCR (Fig. 1), B. parapertussis
DNA was detected in only one sample from hospitalized children. IS481 RT-PCR was positive in 61.5\% (96/156) of the samples from hospitalized children (Fig. 1a). Among these positive samples, $B$. pertussis DNA was specifically detected in $91.7 \%(88 / 96)$ samples, $B$. holmesii DNA was detected in 39.5\%, (38/96) and the coexistence of $B$. pertussis and $B$. holmesii DNA being detected in $36.5 \%$ (35/96) samples while B. holmesii DNA alone was detected only in three samples (Table 1). In 3.1\% (3/96) of the Bordetella positive samples it was not possible to determine the Bordetella species because of the very low amount of DNA.

IS481RT-PCR was positive in $47 \%(59 / 126)$ of the samples from the household contacts. B. pertussis DNA was detected in $90 \%$ (53/59) of these positive samples, and B. holmesii DNA in $18.6 \%$ (11/59), always in samples where $B$. pertussis DNA was already detected. In $8.5 \%(5 / 59)$ of the samples it was not possible to
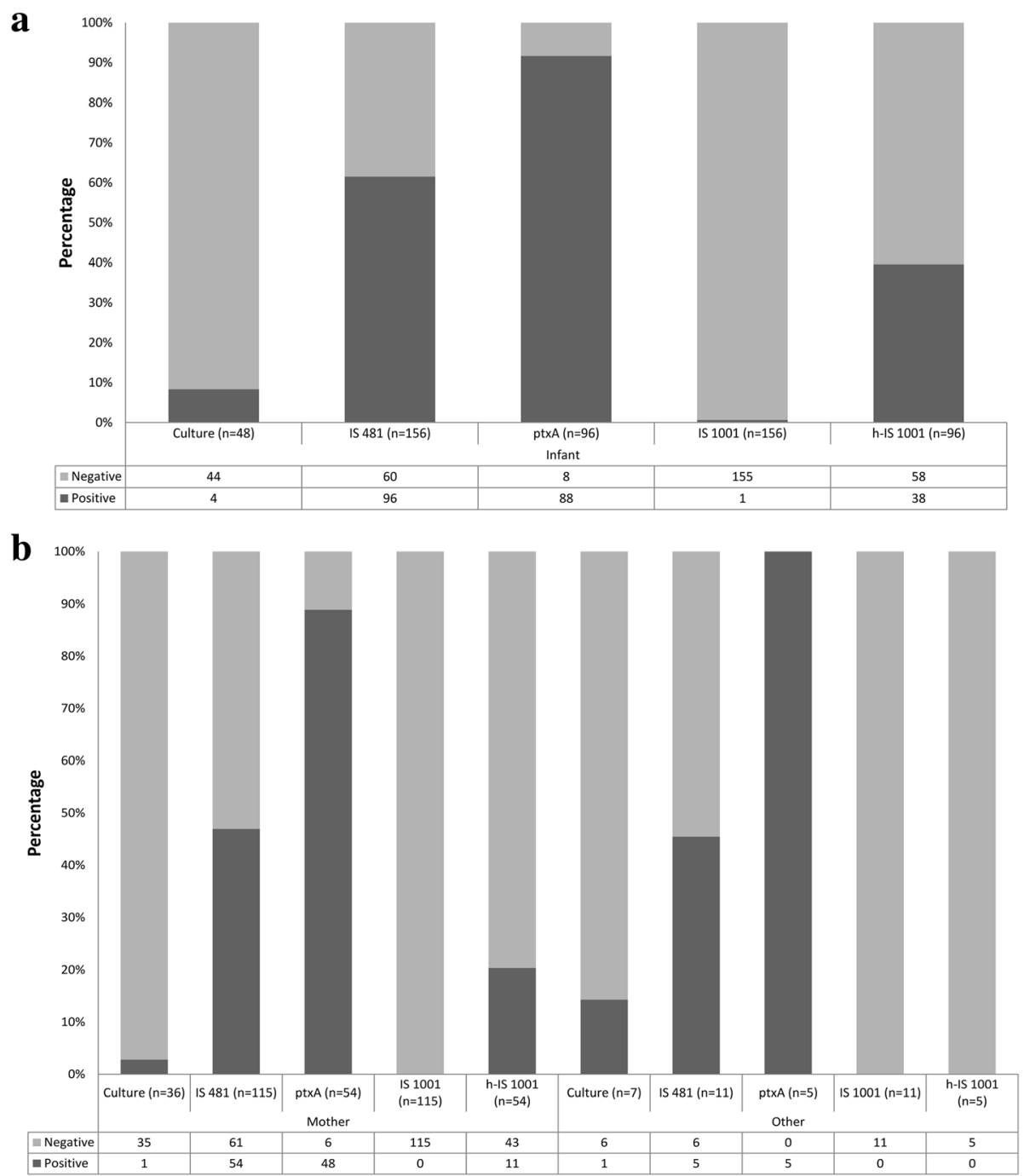

Fig. 1 Pertussis confirmed cases by culture and RT-PCR. a Confirmed cases among hospitalized patients, $\mathbf{b}$ Confirmed cases among contact cases 
Table 1 Results of RT PCR IS481, ptx-Pr and h-IS1001

\begin{tabular}{|c|c|c|c|c|c|c|}
\hline & IS $481+$ & IS 481- & ${ }^{*} p t x+/$ h-IS1001+ & $p t x+/ h-I S 1001-$ & ptx-/h-IS1001+ & $p t x-/ h-I S 1001-$ \\
\hline Patients $(n=156)$ & 96 & 60 & 35 & 55 & 3 & 3 \\
\hline Contacts $(n=126)$ & 59 & 67 & 11 & 42 & 1 & 5 \\
\hline Total & 155 & 127 & 46 & 97 & 4 & 8 \\
\hline
\end{tabular}

ptx: pertussis toxin gene, h-IS1001: $B$. holmesii specific insertion sequence

determine the Bordetella species and they were classified as Bordetella spp.

B. pertussis and B. holmesii DNA were detected in 11 samples from both mothers and siblings. However, $B$. holmesii DNA was also detected in 27 samples from hospitalized children but not in the samples of their mothers. Detection of B. holmesii DNA alone was found in one sample of the entourage.

The common symptoms observed among the 96 confirmed patients were whoops $85.4 \%$ (82/96), cough $68.7 \%$ (66/96) and paroxysmal cough $45.8 \%$ (44/96), or cyanosis $34.3 \%(33 / 96)$, whereas apnea $6.25 \%(6 / 96)$ or post-tussive vomiting $8.3 \%$ (8/96) presented less frequently (Fig. 2).

Our study showed that B. pertussis is also present in healthy people, or with atypical clinical symptoms. Indeed, it has to be noted that 25 confirmed mothers did not declare any clinical symptoms but were in contact to somebody coughing in their household.

It is noteworthy that $89 \%(78 / 88)$ of the confirmed cases had received antibiotic treatment prior to completion of sampling. The administration of Macrolides family antibiotics (Clarithromycin or Azithromycin) was noted in $75 \%$ of them, $15 \%$ received other antibiotics (cefotaxime, ceftriaxone or cotrimoxazole association) and in $10 \%$ of cases, the administered antibiotic was not specified on the information sheet.

The analysis of patients' vaccination status showed that among the 156 hospitalized children enrolled in the study, 61\% (95/156) were unvaccinated and 57\% (89/156) were infants less than two months of age and didn't receive any dose of vaccines according to the Moroccan vaccine strategy recommendation, the other children enrolled $6.3 \%(6 / 95)$ were non vaccinated and were overdue for their first vaccinations between 10 to 30 days. The partially vaccinated patients, $94.2 \%$ (49/52) because of their advanced age, they received only 1 to 2 doses. For other 5.8\% (3/52) the vaccine recommendations were not followed without medical reasons, and especially for the fourth dose (Table 2).

During this surveillance, we met children aged 2238 months who caught whooping cough $(n=3)$ despite they have fulfilled their immunization schedule by vaccinating with 4 doses; other incompletely vaccinated $(n=22)$, with one or two doses were aged between 3 and 14 months.

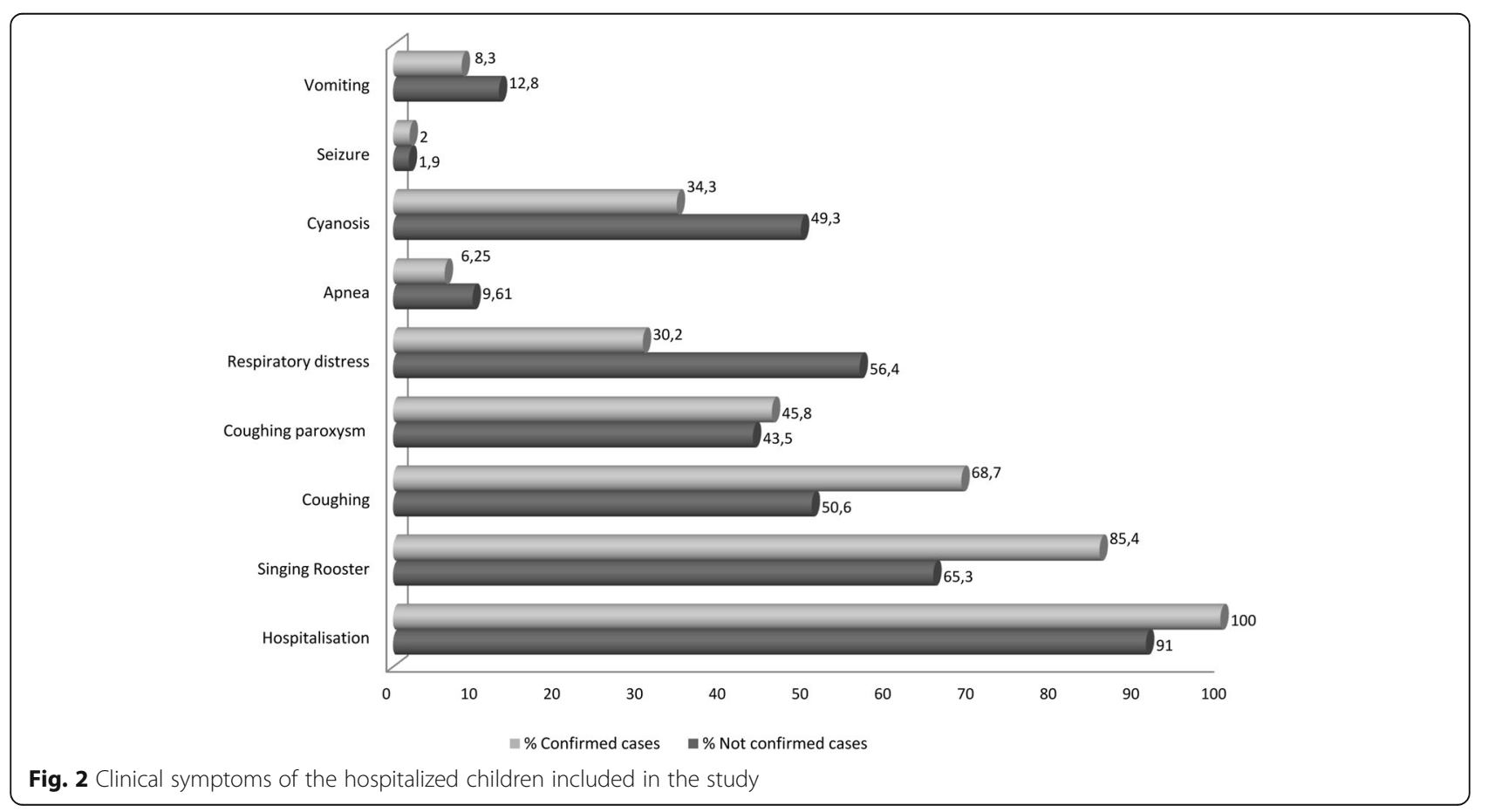


Table 2 PCR results depending on the age and vaccination status

\begin{tabular}{|c|c|c|c|c|}
\hline \multirow[t]{3}{*}{ Vaccination status } & \multicolumn{4}{|c|}{ Age of the patients } \\
\hline & \multicolumn{2}{|c|}{$<2$ months $(n=89)$} & \multicolumn{2}{|c|}{$>2$ months $(n=67)$} \\
\hline & B. holmesii & B. pertussis & B. holmesii & B. pertussis \\
\hline Vaccinated $(n=9)$ & 0 & 0 & 3 & 6 \\
\hline Incompletely vaccinated $(n=52)$ & 0 & 0 & 9 & 43 \\
\hline Non vaccinated $(n=95)$ & 24 & 65 & 2 & 4 \\
\hline Total & 24 & 65 & 14 & 53 \\
\hline
\end{tabular}

\section{Discussion}

Following a significant increase of the number of pertussis cases recorded in many parts of the country from January 2012 (Epidemiological Bulletin, June 2012), we decided to establish a laboratory-based surveillance of pertussis, which discriminates among $B$. pertussis, $B$. parapertussis and B. holmesii.

During 30 months of surveillance, 282 nasopharyngeal samples were collected from patients with clinical suspicions of pertussis disease (156 infants and126 contacts), were received in our laboratory and were analyzed by RT-PCR and Culture. The majority of patients $85 \%$ (132 /156 infants) were admitted in Pediatric Respiratory Diseases service and 15\% were hospitalized in intensive care unit indicating the severity of the disease.

Of the 91 samples cultured, $6(6.5 \%)$ were positive for B. pertussis by biochemical characters identification, very low yield compared to that obtained by RT-PCR (155/282; 55\%).

Culture is the method of choice to isolate the bacteria. However, the chances to obtain positive cultures are high only during the first two weeks of cough and the sensitivity of the method is low as shown in previous studies [7]. In the present study, we collected only 6 isolates for several reasons: (i) 89\% were treated by Clarithromycin or Azithromycin therapy before sampling since several days; (ii) most of the contact patients already coughed since 14 days; (iii) sometime the lack of no fresh medium. All these reasons are causes of low sensitivity of the culture as shown in other studies $[20,21]$. The treatment of choice in the hospital is macrolides and especially Clarithromycin or Azithromycin.

The direct detection of bacterial DNA by PCR techniques especially RT- PCR is more sensitive than culture (up to 4 weeks after the start of coughing fit) and has the advantage of being faster.

In our study the IS 481 RT-PCR confirmed the circulation of Bordetella spp. in the Moroccan population; it infects both unvaccinated infants, teens and adults. To specify Bordetella species in the samples, we made complementary RT-PCR such as RT-PCR Ptx-Pr, IS1001and h-IS1001. The bacterial DNA detected was mostly B. pertussis DNA ( $>90 \%)$ whereas B. parapertussis DNA was only detected in $0.3 \%$ of the samples. The proportion of $B$. parapertussis cases is much lower than in Tunisia where whooping cough was attributed to B. parapertussis in $7 \%$ of cases [22].

RT-PCR h-IS1001 detected B. holmesii DNA in several samples of hospitalized infants (38/96).Surprisingly the large majority of the $B$. holmesii positive samples were also $B$. pertussis positive samples (35/96) indicating coinfection with both bacteria. Furthermore, both bacteria were detected in samples of 11 infants and their mothers suggesting transmission between the mother and the infant but in 27infant cases, the mothers were only infected by $B$. pertussis indicating that the mothers were not the contaminator of $B$. holmesii. The contaminator could have been another member of the household or another reservoir still not known such as animals or the environment. It was already described that the prevalence of $B$. holmesii can widely differ from one region to another even if the regions are geographically close [14] and detected in all age group. This carriage of B. holmesii in infants is different from what was observed in France, Tunisia or US $[15,16,22]$ but similar to what was found in Chile, Romania and Argentina [23-25]. In our study, we identified three patients infected with only B. holmesii; two children lived in slums and the third lived in an orphanage. This leads us to think that the origin of the infection can be animals or environment. Similarly, these children had forms of immunodeficiency (asplenia, chronic obstructive pulmonary disease), they had clinical symptoms compatible with whooping cough but the search for viral coinfection or mycoplasma was not searched.

Misdiagnosis of $B$. holmesii respiratory infection as $B$. pertussis can lead to wrong diagnosis and this affects epidemiological analysis. For this reason it is important to use specific diagnosis even if a little bit less sensitive.In our study there was a high prevalence of coinfections with $B$. pertussis/B. holmesii, as observed in Romania [25]. It is not the case in all recent studies. For example there was no detection in Tunisia [22], detection mainly in adults and in adolescents in France but no co-infection [15] and in US with co-infection or not [16], detection in children in Chile and Argentina $[23,24]$. The isolation or detection of B. holmesii is recent and deserves further studies, in particular on 
the clinical symptoms induced by this Bordetella species. It is difficult in our study to describe $B$. holmesii clinical symptoms since in most of the cases we observed co-infection with $B$. pertussis. B. holmesii DNA alone was detected only in three cases but the search for viral or mycoplasma infection was not searched and the clinical symptoms induced cannot be linked to $B$. holmesii only.

Surveillance and awareness of this species need to be pursued in order to understand its epidemiology [26].

It should be noted that the main objective of vaccine is not only to prevent pertussis in adults but to avoid the contamination of infants too young to be vaccinated by their relatives.

This paradoxical resurgence of whooping cough is not due to solely a progressive loss of immunity in the time or a decrease in immunization coverage in some regions (deletion recall or non-compliance with recommendations), but also be due to lack of vigilance of parents who often slow to vaccinate their children on time, or introducing a new more sensitive laboratory diagnosis such as RT-PCR.

Also, instead of thinking that to prevent infection in early childhood, through the application of booster vaccinations with their entourages (adolescent or adults), we should seek to achieve an early and timely vaccination. WHO propose to initiate at 6 weeks and no later than 8 weeks of age, and maintain high coverage $(\geq 90 \%)$ with at least 3 doses [27].

\section{Conclusion}

We have highlighted the circulation of Bordetella in patients hospitalized at the pediatric hospital by rapid diagnosis based mostly on RT- PCR. The results obtained within this study encourage us to expand it to other laboratories in Morocco in order to improve the biological diagnostic and surveillance of bordetelloses.

In fact we found a high proportion of B. pertussis and B. holmesii co-infection, it is then important to continue the surveillance in order to have a better understanding of the Bordetella spp. circulating in Moroccan population and their role in the respiratory symptoms.

Moreover, our study suggests that infection of nonvaccinated infants is associated with parents, hence the need to investigate around the index cases to detect contaminators and prevent contamination of young infants and disease spread by quick treatment. Furthermore, the RT- PCR provides a sensitive and specific diagnosis of $B$. pertussis infections and distinguishes it from other Bordetella species, and is therefore suitable for implementation in the diagnostic laboratory.

\section{Abbreviations}

ptx: pertussis toxin gene; RT-PCR: real time polymerase chain reaction; WHO: World Health Organization

\section{Acknowledgements}

We would like to acknowledge Dr. Sophie Guillot and colleagues of the French National Reference Center of Whooping Cough and Other Bordetelloses at the Institute Pasteur of Paris for provision of laboratory protocols and for providing reference strains. We also thank all the clinicians for sending samples for analysis.

\section{Funding}

This work was supported by an unrestricted grant of SANOFI-PASTEUR laboratory. The authors conceived the study, and the study design was developed and agreed to by the authors without any input from the funding body. The funding body was not involved in and, had no influence over, study design, data collection, data analyses, interpretation of results, report writing or in the decision to submit the paper for publication.

\section{Availability of data and materials}

The datasets used and/or analysed during the current study are available from the corresponding author on reasonable request.

\section{Authors' contributions}

$\mathrm{NE}, \mathrm{KK}, \mathrm{KZ}, \mathrm{HB}$ and $\mathrm{NG}$ conceived and designed the study. KK, BS, ZJ and AZ conducted the case surveillance and collected clinical data. ID and KK conducted the laboratory assays. NG, NE, ID and KK analyzed the data. NG, $\mathrm{NE}, \mathrm{KK}, \mathrm{ID}$ and $\mathrm{KZ}$ drafted the manuscript. All authors read and approved the final manuscript.

\section{Competing interests}

The authors declare that they have no competing interests.

\section{Consent for publication}

Not applicable.

\section{Ethics approval and consent to participate}

This study was approved by the Ethical committee for biomedical research of the University Mohammed V - Soussi, Faculty of Medicine, Pharmacy and Dental Medicine of Rabat, Morocco N77/16. The study was anonymous and all patients and household contacts, and their parents/guardians for minors below 18 years of age, were informed by their physicians about the nature of the study. Only patients who agreed to participate in this study signed the informed consent. The fact that samples will be stored for a certain period of time before testing was clearly indicated in the inform consent statement.

\section{Publisher's Note}

Springer Nature remains neutral with regard to jurisdictional claims in published maps and institutional affiliations.

\section{Author details}

${ }^{1}$ Laboratoire de Microbiologie, Faculté de Médecine et de Pharmacie, Hassan II University of Casablanca, B, P 5696 Casablanca, Morocco. ${ }^{2}$ Service de Microbiologie, CHU Ibn Rochd, B, P 2698 Casablanca, Morocco. ${ }^{3}$ Molecular Prevention and Therapy of Human Diseases, Institut Pasteur, 25 rue du Dr Roux, 75015 Paris, France. ${ }^{4}$ Service des Maladies Respiratoires Pédiatriques, Hôpital d'Enfants Abderrahim Harouchi, CHU Ibn Rochd de Casablanca, Casablanca, Morocco. ${ }^{5}$ Service des Maladies Infectieuses Pédiatriques, Hôpital d'Enfants Abderrahim Harouchi, CHU Ibn Rochd de Casablanca, Casablanca, Morocco. ${ }^{6}$ Service d'Accueil des Urgences Pédiatriques, Hôpital d'Enfants Abderrahim Harouchi, CHU Ibn Rochd de Casablanca, Casablanca, Morocco. ${ }^{7}$ Bacteriology-Virology and Hospital Hygiene Laboratory, University Hospital Centre Ibn Rochd, 1, Rue des Hôpitaux, 20100 Casablanca, Morocco.

Received: 26 December 2016 Accepted: 8 May 2017

Published online: 16 May 2017

\section{References}

1. World Health Organization. Immunizations, Vaccines and Biologicals: Pertussis. 2011. Accessed Nov 2016. [http://www.who.int/immunization/ topics/pertussis/en].

2. Zepp F, Heininger U, Mertsola J, et al. Rationale for pertussis booster vaccination throughout life in Europe. Lancet Infect Dis. 2011;11:557-70. 
3. WHO. Revised guidance on the choice of pertussis vaccines: July 2014. Wkly Epidemiol Rec. 2014;89:337-340.

4. Ouardirhi A. Vaccination contre la coqueluche : Le moyen de prévention le plus efficace. Albayane. 2012;

5. Royaume du Maroc - Ministère de la Santé. Bulletin Epidémiologique. 2012.

6. Cherry JD, Grimprel E, Guiso N, Heininger U, Mertsola J. Defining pertussis epidemiology: clinical, microbiologic and serologic perspectives. Pediatr Infect Dis J. 2005;24:S25-34.

7. Wirsing von Konig $\mathrm{CH}$. Pertussis diagnostics: overview and impact of immunization. Expert review of vaccines. 2014;13:1167-74.

8. World Health Organization. Laboratory Manual for the diagnosis of whooping cough caused by bordetella pertussi. 2014.

9. Nakamura Y, Kamachi K, Toyoizumi-Ajisaka H, et al. Marked difference between adults and children in Bordetella pertussis DNA load in nasopharyngeal swabs. Clin Microbiol Infect. 2011:17:365-70.

10. Riffelmann M, Wirsing von Konig CH, Caro V, Guiso N. Nucleic acid amplification tests for diagnosis of Bordetella infections. J Clin Microbiol. 2005;43:4925-9.

11. Tizolova A, Guiso N, Guillot S. Insertion sequences shared by Bordetella species and implications for the biological diagnosis of pertussis syndrome. Eur J Clin Microbiol Infect Dis. 2013;32:89-96.

12. Shepard CW, Daneshvar Ml, Kaiser RM, et al. Bordetella holmesii bacteremia: a newly recognized clinical entity among asplenic patients. Clin Infect Dis. 2004;38:799-804.

13. Yih WK, Silva EA, Ida J, et al. Bordetella holmesii-like organisms isolated from Massachusetts patients with pertussis-like symptoms. Emerg Infect Dis. 1999;5:441-3.

14. Pittet LF, Emonet S, Schrenzel J, Siegrist CA, Posfay-Barbe KM. Bordetella holmesii: an under-recognised Bordetella species. Lancet Infect Dis. 2014:14:510-9.

15. Njamkepo E, Bonacorsi S, Debruyne M, et al. Significant finding of Bordetella holmesii DNA in nasopharyngeal samples from French patients with suspected pertussis. J Clin Microbiol. 2011;49:4347-8.

16. Rodgers L, Martin SW, Cohn A, et al. Epidemiologic and laboratory features of a large outbreak of pertussis-like illnesses associated with cocirculating Bordetella holmesii and Bordetella pertussis-Ohio, 2010-2011. Clin Infect Dis. 2013:56:322-31.

17. André P, Caro V, Njamkepo E, et al. Comparison of serological and real-time PCR assays to diagnose Bordetella pertussis infection in 2007. J Clin Microbiol. 2008:46:1672-7.

18. Tatti KM, Sparks KN, Boney KO, Tondella ML. Novel multitarget real-time PCR assay for rapid detection of Bordetella species in clinical specimens. J Clin Microbiol. 2011;49:4059-66.

19. Guillot S, Guiso N. Follow-up of external quality controls for PCR-based diagnosis of whooping cough in a hospital laboratory network (Renacoq) and in other hospital and private Laboratories in France. J Clin Microbiol. 2016;54:2169-71

20. Bidet P, Liguori S, De Lauzanne A, et al. Real-time PCR measurement of persistence of Bordetella pertussis DNA in nasopharyngeal secretions during antibiotic treatment of young children with pertussis. J Clin Microbiol. 2008:46:3636-8.

21. Guiso N. Bordetella pertussis: why is it still circulating? I Inf Secur. 2014;68(Suppl 1):S119-24.

22. Zouari A, Smaoui H, Brun D, et al. Prevalence of Bordetella pertussis and Bordetella parapertussis infections in Tunisian hospitalized infants: results of a 4-year prospective study. Diagn Microbiol Infect Dis. 2012;72:303-17.

23. Bottero D, Griffith MM, Lara C, et al. Bordetella holmesii in children suspected of pertussis in Argentina. Epidemiol Infect. 2013;141:714-7.

24. Miranda C, Porte L, Garcia P. Bordetella holmesii in nasopharyngeal samples from Chilean patients with suspected Bordetella pertussis infection. J Clin Microbiol. 2012:50:1505. author reply 1506

25. Dinu S, Guillot S, Dragomirescu CC, et al. Whooping cough in south-East Romania: a 1-year study. Diagn Microbiol Infect Dis. 2014;78:302-6.

26. Diavatopoulos DA, Cummings CA, van der Heide HG, et al. Characterization of a highly conserved island in the otherwise divergent Bordetella holmesii and Bordetella pertussis genomes. J Bacteriol. 2006;188:8385-94.

27. WHO. Pertussis vaccines: WHO position paper, August 2015Recommendations. Vaccine. 2016:34:1423-1425.

\section{Submit your next manuscript to BioMed Central and we will help you at every step:}

- We accept pre-submission inquiries

- Our selector tool helps you to find the most relevant journal

- We provide round the clock customer support

- Convenient online submission

- Thorough peer review

- Inclusion in PubMed and all major indexing services

- Maximum visibility for your research

Submit your manuscript at www.biomedcentral.com/submit
Biomed Central 